\title{
Circular RNA UBAP2 promotes the proliferation of prostate cancer cells via the miR-1244/MAP3K2 axis
}

\author{
XIAODONG LI, BAIHETIYA AZHATI, WENGUANG WANG, MULATI REXIATI, CHEN XING and YUJIE WANG \\ Department of Urology, The First Affiliated Hospital of Xinjiang Medical University, \\ Urumqi, Xinjiang Uygur Autonomous Region 830054, P.R. China
}

Received October 11, 2020; Accepted February 25, 2021

DOI: $10.3892 / \mathrm{ol} .2021 .12747$

\begin{abstract}
Prostate cancer (PCa) is a common male malignant disease with a high incidence, which can seriously affect the quality of life of patients. The survival rate of patients with PCa has improved to $98.6 \%$; however, new insights for the molecular mechanism are still urgently required. Circular RNA (circ)UBAP2 is a tumor-associated circRNA that has been demonstrated to promote the progression of various types of cancer. CircUBAP2 has been demonstrated to be significantly upregulated in $\mathrm{PCa}$, but its role in the progression of PCa remains unclear. The present study aimed to provide an improved understanding of the regulatory mechanism of circUBAP2 in PCa. circUBAP2 expression was identified to be upregulated in four PCa cell lines and clinical tissues by using reverse transcription-quantitative PCR analysis. Binding sites analysis and luciferase reporter gene assay indicated that the microRNA(miR)-1244/MAP3K2 axis was the target of circUBAP2. Gain-of-function assays revealed that circUBAP2 promoted the proliferation of PCa cells by sponging miR-1244 and promoting the MAP3K2 axis. The present findings may be essential for providing new strategies in the diagnosis and targeted therapy of PCa.
\end{abstract}

\section{Introduction}

Prostate cancer ( $\mathrm{PCa})$ is one of the most common types of malignant cancer of the prostatic epithelium $(1,2)$. Age and heredity are the main risk factors associated with PCa development (3). The age of onset ranges from 65 to 74 years, with a median age of 66 years (4). It is estimated that $>1,600,000$ new cases are diagnosed annually (5). The incidence of PCa is significantly higher in the Western population than in the

Correspondence to: Dr Yujie Wang, Department of Urology, The First Affiliated Hospital of Xinjiang Medical University, 137 Liyushan South Road, Urumqi, Xinjiang Uygur Autonomous Region 830054, P.R. China

E-mail: wyj2018vip@126.com

Keywords: prostate cancer, circular RNA UBAP2, microRNA-1244, MAP3K2, MAPK signaling pathway, proliferation
Asian one (6). In the United States, $\sim 1$ out of 5 men will be diagnosed with $\mathrm{PCa}$ (4), while in China, the incidence of PCa is 7.1/100,000 individuals (7). Although PCa has a 5-year survival rate of $98.6 \%(4,8)$, an in-depth understanding of its pathogenesis and new therapeutic strategies are still urgently required.

Circular RNAs (circRNAs), a large class of non-coding RNAs, have attracted increasing attention over the last decade (9). Preliminary studies have revealed that circRNAs serve an important role in post-transcriptional regulation by acting as microRNA (miRNA/miR) sponges or by encoding peptides (10-12). CircRNAs have been reported to have unique expression profiles in different cancer subtypes and they regulate multiple biological processes, such as development, differentiation, apoptosis and cell proliferation (13). Some circRNAs have already been considered as tumor-associated circRNAs, such as CDR1as, circUBAP2 and circPTV1 (14). Among these, circUBAP2 has been reported as a critical regulator involved in the progression of several types of cancer, such as ovarian cancer, esophageal squamous cell carcinoma and pancreatic adenocarcinoma (15-17).

Recently, Chen et al (18) revealed the circRNA transcriptional landscape with functional annotation in localized PCa. In their circRNA profile of PCa, circUBAP2 was significantly abundantly expressed in $\mathrm{PCa}$ (18). However, the role of circUBAP2 in the progression of PCa remains unclear. Hence, the present study aimed to clarify the regulatory mechanism of circUBAP2 in the progression of PCa.

\section{Materials and methods}

Cell culture. Four human PCa cell lines (LNCaP, V16A, DU145 and PC-3) were purchased from the American Type Culture Collection (ATCC) and cultured in RPMI 1640 medium (Gibco; Thermo Fisher Scientific Inc.) with $10 \%$ fetal bovine serum (FBS; Thermo Fisher Scientific Inc.) and $1 \%$ penicillin-streptomycin $(18,19)$. Human prostate epithelial cell line (RWPE-1) was purchased from ATCC and cultured in Keratinocyte serum-free medium (KSFM; Thermo Fisher Scientific Inc.) (20). All cells were maintained in a $37^{\circ} \mathrm{C}$ humidified atmosphere with $5 \% \mathrm{CO}_{2}$.

Clinical tissues. A total of 20 male patients who were diagnosed as prostate cancer and underwent prostatectomy at The First 
Affiliated Hospital of Xinjiang Medical University (Urumqi, China) from January 2020 to March 2020 were included in the present study. Patients with immunological diseases, metabolic diseases, hypertension diabetes or any other type of tumor diseases were excluded. The mean age of enrolled patients was $74.38 \pm 3.32$ years (age range, $68-81$ years). $P C a$ and paired adjacent normal tissues ( $2 \mathrm{~cm}$ distance from tumor tissue) were obtained after prostatectomy and stored in liquid nitrogen. Written informed consent was obtained from each participant. All study protocols were approved by the Ethics Committee of The First Affiliated Hospital of Xinjiang Medical University (approval no. XJ20200025; Urumqi, China).

Reverse transcription-quantitative (RT-q) PCR. Total RNA from PCa cell lines and tissues was extracted using TRIzol ${ }^{\circledR}$ reagent (Invitrogen; Thermo Fisher Scientific Inc.). Nuclear and cytoplasmic RNA were separated using a Cytoplasmic and Nuclear RNA Purification kit (Norgen Biotek Corp.). The miRNAs were reverse transcribed to cDNA using the All-in-one ${ }^{\mathrm{TM}}$ miRNA First-Strand cDNA Synthesis kit (GeneCopoeia Inc.) and the mRNAs were reverse transcribed to cDNA using the Oligo(dT) priming method (GeneCopoeia Inc.) according to the manufacturer's protocol. SYBR Green qPCR Master Mix (Thermo Fisher Scientific Inc.) was used to detect the expression levels of miRNAs and mRNAs via qPCR. The qPCR thermocycling conditions were as follows: Enzyme activation at $95^{\circ} \mathrm{C}$ for $30 \mathrm{sec}$, followed by 40 two-step cycles $\left(95^{\circ} \mathrm{C}\right.$ for $5 \mathrm{sec} ; 60^{\circ} \mathrm{C}$ for $\left.30 \mathrm{sec}\right)$. The related expression levels of miRNAs and mRNAs were normalized to U6 and GAPDH, respectively, according to the $2^{-\triangle \Delta C q}$ method (21).

The primers were as follows: circUBAP2 forward, 5'-AGC CTCAGAAGCCAACTCCTTTG-3' and reverse, 5'-TCAGGT TGAGATTTGAAGTCAAGAT-3'; MAP3K2 forward, 5'-CCC CAGGTTACATTCCAGATGA-3' and reverse, 5'-GCATTC GTGATTTTGGATAGCTC-3'; ERK1 forward, 5'-TACACC AACCTCTCGTACATCG-3' and reverse, 5'-CATGTCTGA AGCGCAGTAAGATT-3'; JNK forward, 5'-TGTGTGGAA TCAAGCACCTTC-3' and reverse, 5'-AGGCGTCATCAT AAAACTCGTTC-3'; p53 forward, 5'-CAGCACATGACG GAGGTTGT-3' and reverse, 5'-TCATCCAAATACTCCACA CGC3'; GAPDH forward, 5'-ACCACAGTCCATGCCATC AC-3' and reverse, 5'-TCCACCACCCTGTTGCTGTA-3'; miR-1244 forward, 5'-ACACTCCAGCTGGGAAGTAGT TGGTTTGTATGAG-3' and reverse, 5'-CTCAACTGGTGT CGTGGAGTCGGCAATTCAGTTGAGAACCATCT-3'; and U6 forward, 5'-AAAGCAAATCATCGGACGACC-3' and reverse, 5'-GTACAACACATTGTTTCCTCGGA-3'.

miRNA and mRNA expression profiles analysis. The expression profiles of miRNA (GSE76260) and mRNA (GSE30994) were downloaded from the Gene Expression Omnibus (GEO) database $(22,23)$. The differentially expressed miRNAs and mRNAs were detected using the GEO2R tool (https://www. ncbi.nlm.nih.gov/geo/geo2r), which allowed users to compare two or more groups of samples in a GEO Series. After eliminating duplicates, |fold-change (FC) $\mid \geq 2$ and adjusted $\mathrm{P}<0.05$ were set as the criteria for differentially expressed miRNAs.

Competitive endogenous RNA (ceRNA) network. The circUBAP2-targeted miRNAs were predicted using circBank (circbank.cn) and circular RNA interactome (circinteractome.nia.nih.gov) (24). The miRNAs predicted by both of these websites were considered as potential target miRNAs of circUBAP2. The expression levels of these potential target miRNAs in PCa were further confirmed based on the GSE76260 miRNA expression profile. The potential target mRNAs were screened out using miRWalk (mirwalk.umm. uni), TargetScan (targetscan.org), miRDB (mirdb.org) and TargetMiner (isical.ac.in) (25). Their expression levels in PCa were also validated based on the GSE30994 mRNA expression profile. The circUBAP2-associated ceRNA network was constructed using Cytoscape (https://cytoscape.org/).

Inhibition of miR-1244 and circUBAP2. The miR-1244 inhibitor (the sequences were not provided) and scrambled negative control (the sequences were not provided) was designed and synthesized by Suzhou GenePharma Co., Ltd. The sequence of circUBAP2 was obtained using circBase (http://www.circbase. org/). The small interfering (si)RNA targeting circUBAP2 (si-circUBAP2; siRNA-1, 5'-GCTTCTAAGCTTTCTGAA ACA-3'; siRNA-2, 5'-CAGCTTCTAAGCTTTCTGAAA-3'; and siRNA-3, 5'-CCCAGCTTCTAAGCTTTCTGA-3') and scrambled negative control (si-control; the sequences were not provided) were also designed and synthesized by Suzhou GenePharma Co., Ltd. (26).

Cell transfection. The miR-1244 inhibitor or si-circUBAP2 (both $100 \mathrm{nM}$ ) were transfected into PCa cells using Lipofectamine ${ }^{\circledR} 3000$ (Thermo Fisher Scientific Inc.) and then the cells were maintained in a $37^{\circ} \mathrm{C}$ humidified atmosphere with $5 \% \mathrm{CO}_{2}$ for $6 \mathrm{~h}$, following which culture medium was replaced. After $48 \mathrm{~h}$, RT-qPCR was performed to detect the mRNA expression levels.

Luciferase reporter gene assay. The luciferase reporter gene plasmid pGL6-miR (Beyotime Institute of Biotechnology) containing the putative binding site of miR-1244 was constructed and validated by Sangon Biotech Co., Ltd. Human embryonic kidnFS (293T) cells were purchased from the American Type Culture Collection (ATCC) and cultured in Dulbecco's modified Eagle's medium (DMEM; Thermo Fisher Scientific, Inc.) with $10 \%$ fetal bovine serum (FBS; Gibco; Thermo Fisher Scientific, Inc.) and 1\% penicillin-streptomycin. Subsequently, the 293T cells were seeded into a 24 -well plate at a density of $2 \times 10^{5}$ cells/well and then cultured at $37^{\circ} \mathrm{C}$ with $5 \% \mathrm{CO}_{2}$ and $95 \%$ humidity. $293 \mathrm{~T}$ cells were transfected with pGL6-miR with or without miR-1244 mimics or NC (Suzhou GenePharma Co., Ltd.) using Lipofectamine 3000. Luciferase protein was stained via immunofluorescence according to the manufacturer's instructions. The luciferase activity was measured $48 \mathrm{~h}$ after transfection with a Dual-Luciferase Reporter Assay System (Promega Corporation) using a fluorescence spectrophotometer (Infinite M200; Tecan Group, Ltd.), which was then normalized to Renilla luciferase activity.

Western blotting. Total protein from PCa cells were extracted by using the Total Protein Extraction kit (cat. no. PE001; Signalway Antibody LLC) and quantified using the bicinchoninic acid (BCA) protein quantitative method. The proteins were subjected to $10 \%$ SDS-PAGE with equal amounts 

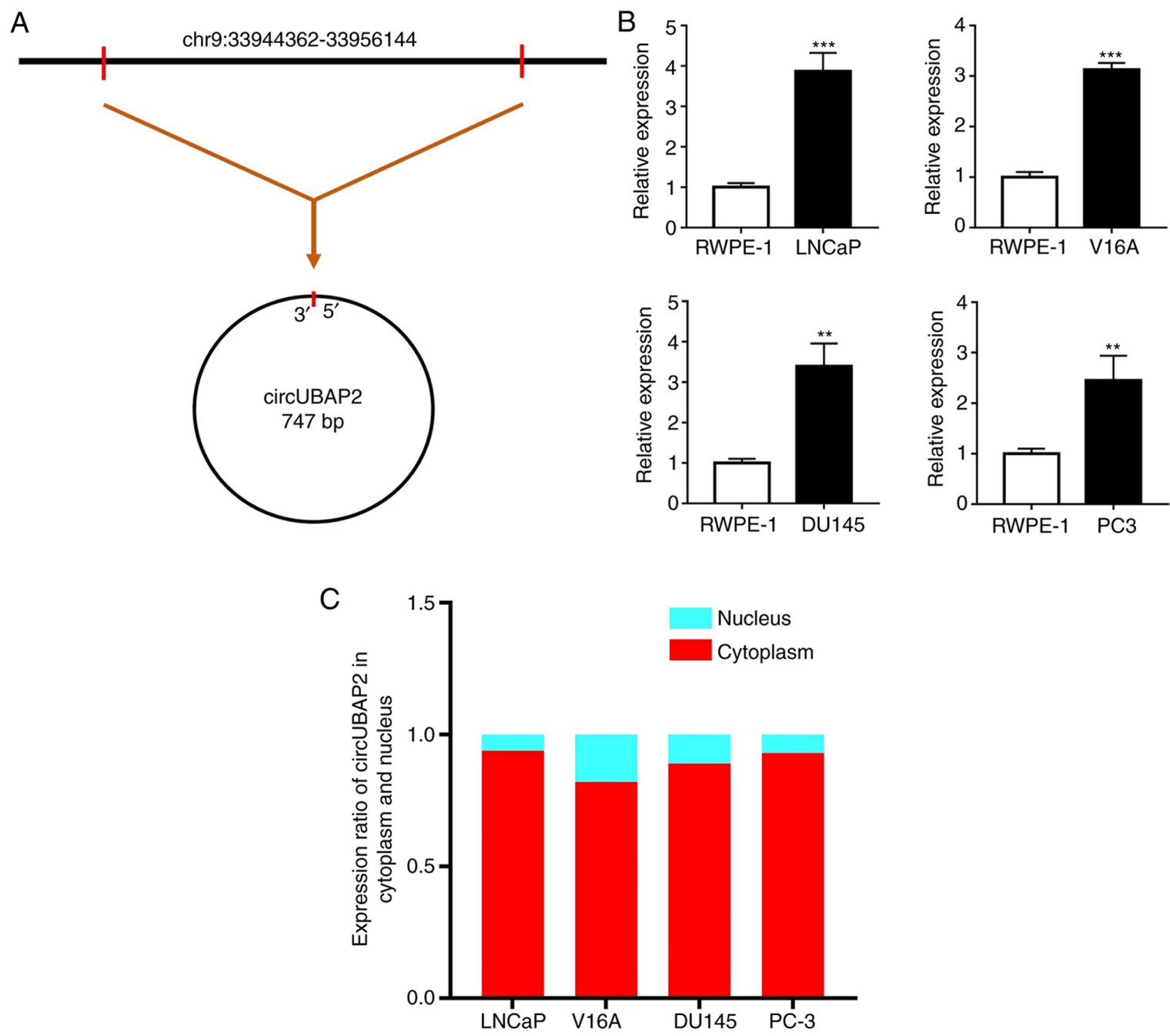

Figure 1. Validation of circUBAP2 in PCa cell lines. (A) Genomic position of circUBAP2. (B) circUBAP2 expression was upregulated in four human PCa cell lines (LNCaP, V16A, DU145 and PC-3). (C) circUBAP2 was mainly located in the cytoplasm of PCa cell lines. ${ }^{* *} \mathrm{P}<0.01$ and ${ }^{* * * *} \mathrm{P}<0.001 \mathrm{vs.} \mathrm{RWPE}-1$ cells. PCa, prostate cancer; circUBAP2, circular RNA UBAP2.

(50 $\mu \mathrm{g} /$ lane) and then transferred onto PVDF membranes. After blocking with 5\% skimmed milk for $1 \mathrm{~h}$ at room temperature, membranes were washed by TBST with $0.5 \%$ Tween 205 times and incubated on a plate shaker with primary antibodies overnight at $4^{\circ} \mathrm{C}$. Subsequently, membranes were washed again and probed by secondary antibodies for $1 \mathrm{~h}$ at room temperature. Finally, the protein bands were visualized using an ECL luminescence reagent (Sangon Biotech Co., Ltd.). Primary antibodies against MAP3K2 (1:10,000; cat. no. ab240926), ERK (1:1,000; cat. no. ab32081), JNK (1:1,000; cat. no. ab110724), p38 (1:1,000; cat. no. ab170099), $\beta$-actin (1:5,000; cat. no. ab179467) and secondary horse-radish peroxidase conjugated antibodies (goat anti-rabbit IgG; 1:5,000, cat. no. ab7090) used in the present study were all purchased from Abcam. $\beta$-actin was used as the loading control.

Cell counting kit-8 (CCK-8) assay. CCK-8 assay was performed to evaluate the effects of circUBAP2-knockdown on the proliferation of PCa LNCaP, V16A, DU145 and PC-3 cell lines. The PCa cells were seeded into 96-well plates and cultured at $37^{\circ} \mathrm{C}$. Subsequently, the CCK-8 solution ( $10 \mu \mathrm{l} /$ well $)$ (Beyotime Institute of Biotechnology) was added for $2 \mathrm{~h}$ at each time point (48, 96 and $144 \mathrm{~h}$ post-transfection). The cell proliferative activity was reflected by measuring the optical density at a wavelength of $450 \mathrm{~nm}$ using Multiskan Spectrum (Thermo Fisher Scientific Inc.).

Statistical analysis. The $\mid \mathrm{FC} \mathrm{I} \geq 2$ and adjusted $\mathrm{P}<0.05$ indicated that miRNA and mRNA were significantly differentially expressed. The data of RT-qPCR and CCK- 8 assays were expressed as the mean \pm SD of 3 repeats. The paired Student's t-test was used to compare the differences between two groups. One-way ANOVA (parametric) was used to compare the differences among multiple groups, with Tukey's test as the post-hoc test. The correlation analysis was determined using Pearson's correlation coefficient. Statistical analysis was performed using GraphPad Prism 7.0 (GraphPad Software, Inc.). $\mathrm{P}<0.05$ was considered to indicate a statistically significant difference.

\section{Result}

circUBAP2 is highly expressed in four PCa cell lines. According to circBase, circUBAP2 is generated from the 


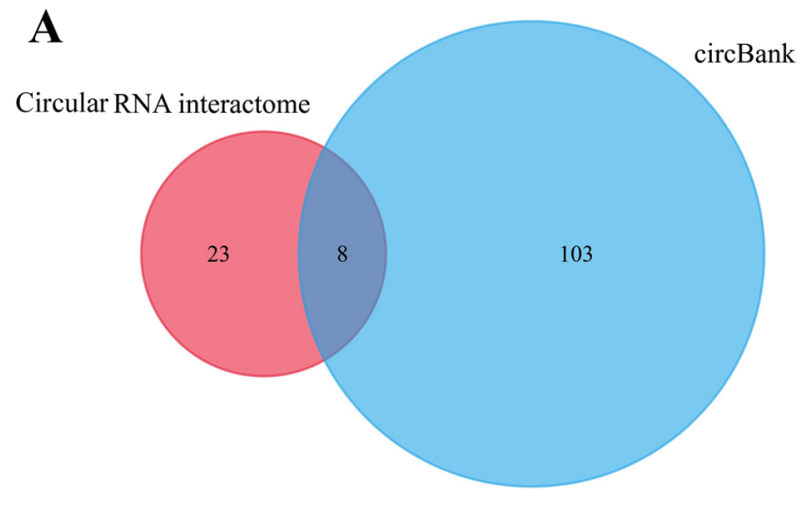

C

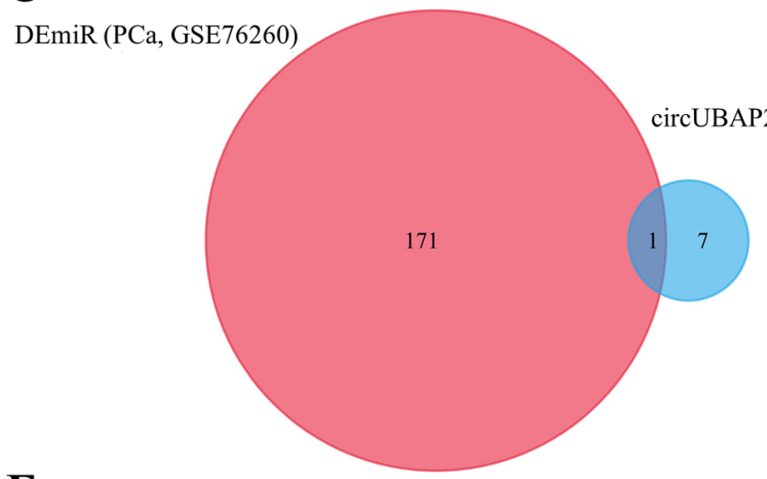

$\mathbf{E}$

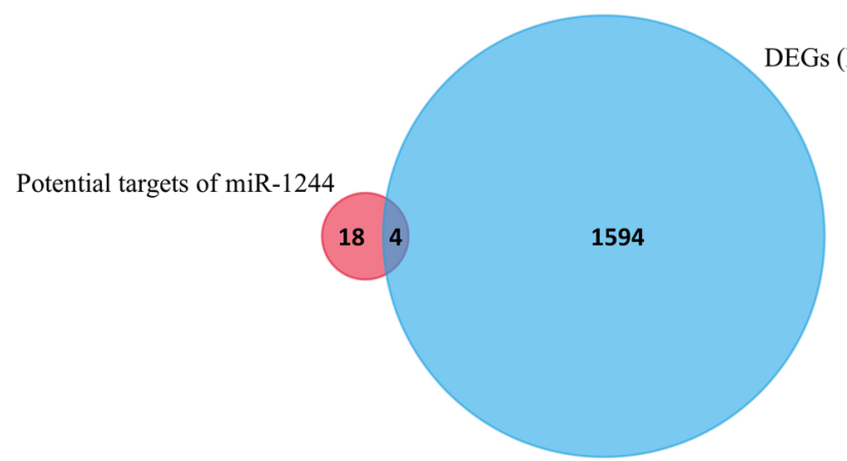

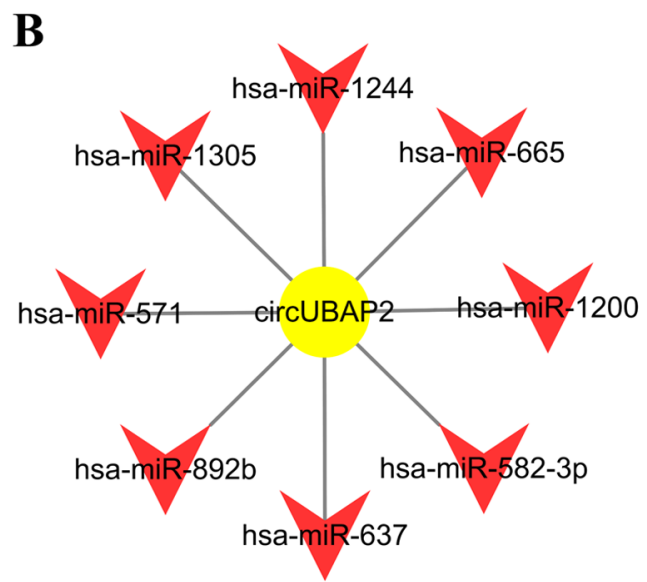

D

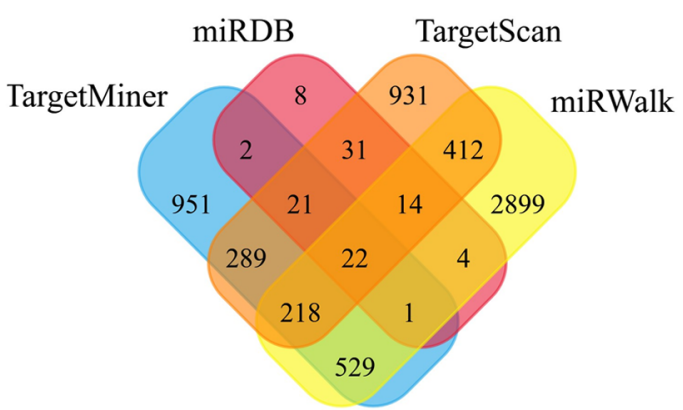

F

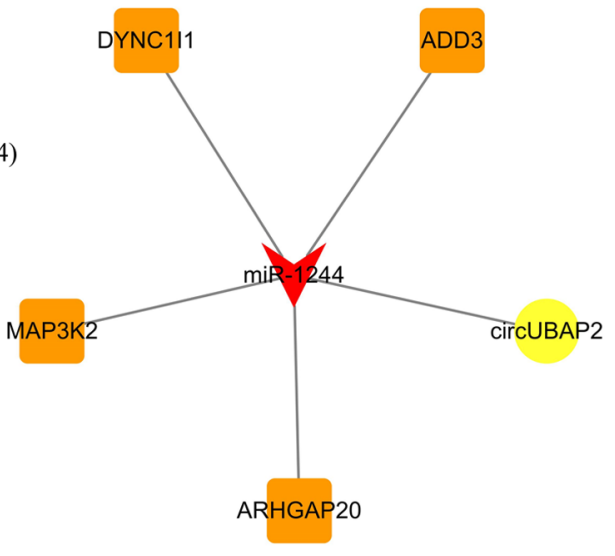

Figure 2. Prediction of the circUBAP2-associated axis. (A) A total of 31 and 111 miRNAs were predicted as targets of circUBAP2 using circular RNA interactome and circBank, respectively, and 8 of these miRNAs were co-predicted. (B) The potential regulatory network of circUBAP2 and its targets miR-1244, miR-665, miR-1200, miR-582-3p, miR-637, miR-892b, miR-571 and miR-1305. (C) miR-1244 was the only DEmiR in the miRNA profile GSE76260. (D) A total of 22 mRNAs were predicted as the potential target mRNAs of miR-1244, which were co-predicted using miRDB, TargetScan, miRWalk and TargetMiner. (E) Four co-predicted DEGs (ADD3, DYNC1I1, MAP3K2 and ARHGAP20) in the mRNA expression profile GSE30994. (F) circUBAP2-associated competitive endogenous RNA network. circUBAP2, circular RNA UBAP2; DE, differentially expressed; DEGs, DE genes; miR/miRNA, microRNA; ADD3, adducin 3; DYNC1I1, dynein cytoplasmic 1 intermediate chain 1; ARHGAP20, Rho GTPase activating protein 20; PCa, prostate cancer.

UBAP2 gene and its genomic position, as shown in Fig. 1A. The expression levels of circUBAP2 were detected in four human PCa cell lines (LNCaP, V16A, DU145 and PC-3) and in a human normal prostate cell line (RWPE-1). The results revealed that circUBAP2 expression was significantly upregulated in all four PCa cell lines compared with in RWPE-1 cells (Fig. 1B). Among these PCa cell lines, LNCaP exhibited the highest expression levels of circUBAP2. Additionally, the expression levels of circUBAP2 were further validated in the nucleus and cytoplasm of PCa cell lines separately to confirm its location in PCa cells. The results indicated that circUBAP2 was mainly expressed in the cytoplasm, which was important for its function prediction (Fig. 1C).

Potential targets of circUBAP2. To further explore the regulatory effect of circUBAP2 in $\mathrm{PCa}$ cells, its target miRNAs were predicted using circular RNA interactome and circBank. Venn diagram analysis was further used to identify the common miRNAs in both websites (Fig. 2A). The results indicated that miR-1244, miR-665, miR-1200, miR-582-3p, miR-637, miR-892b, miR-571 and miR-1305 were the potential target miRNAs of circUBAP2 (Fig. 2B). Their expression levels in PCa cells were then validated 
A

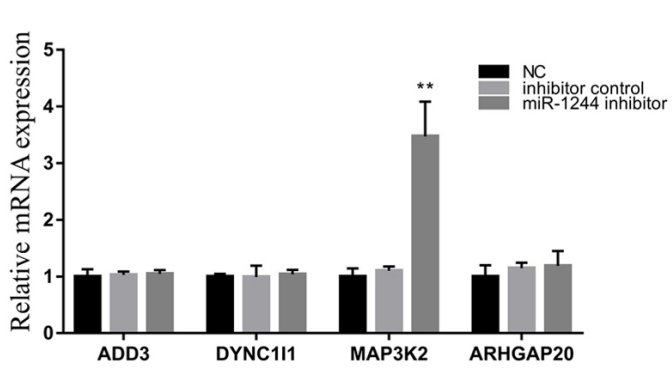

B

\begin{tabular}{|c|c|}
\hline Target region of miR-1244 & Target type \\
\hline 1669-1676 of MAP3K2 3' UTR & $8 \mathrm{mer}$ \\
\hline 2552-2559 of MAP3K2 3' UTR & $8 \mathrm{mer}$ \\
\hline 2752-2759 of MAP3K2 3' UTR & $8 \mathrm{mer}$ \\
\hline $411-418$ of circUBAP2 & $8 \mathrm{mer}$ \\
\hline
\end{tabular}

C

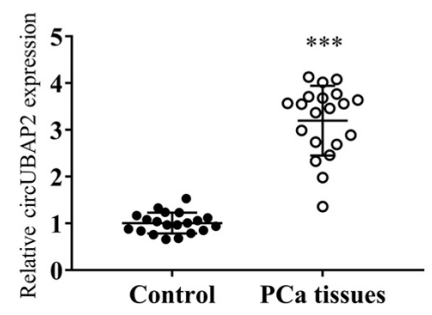

D

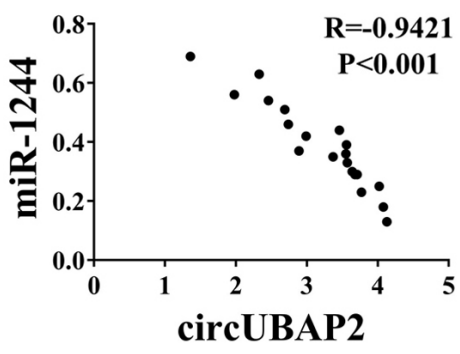

$\mathbf{E}$
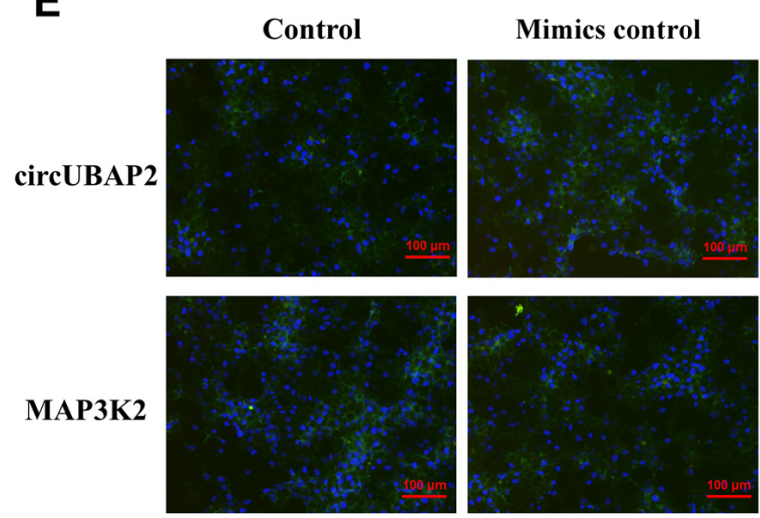

MAP3K2

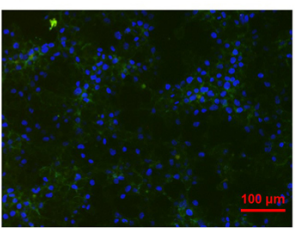

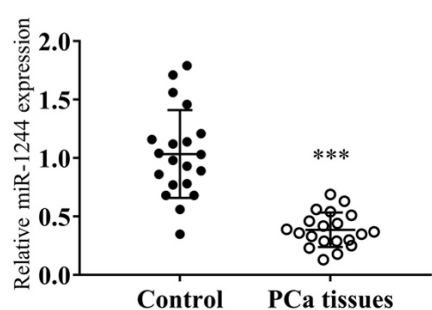

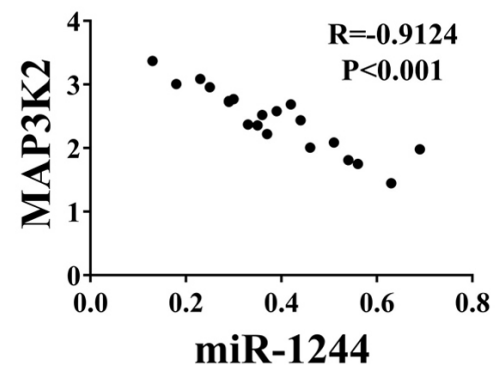

F
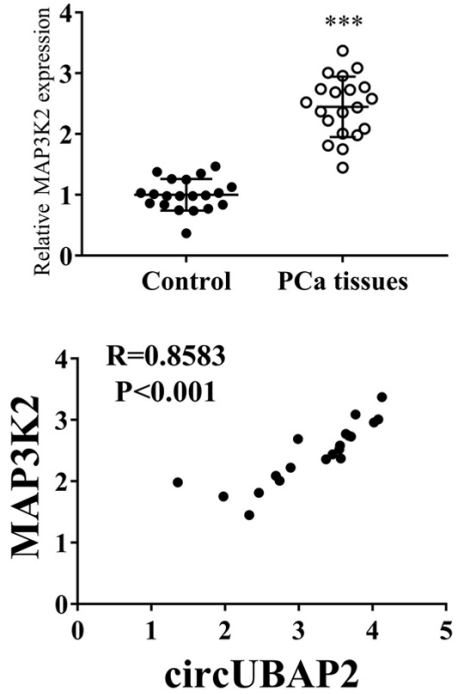
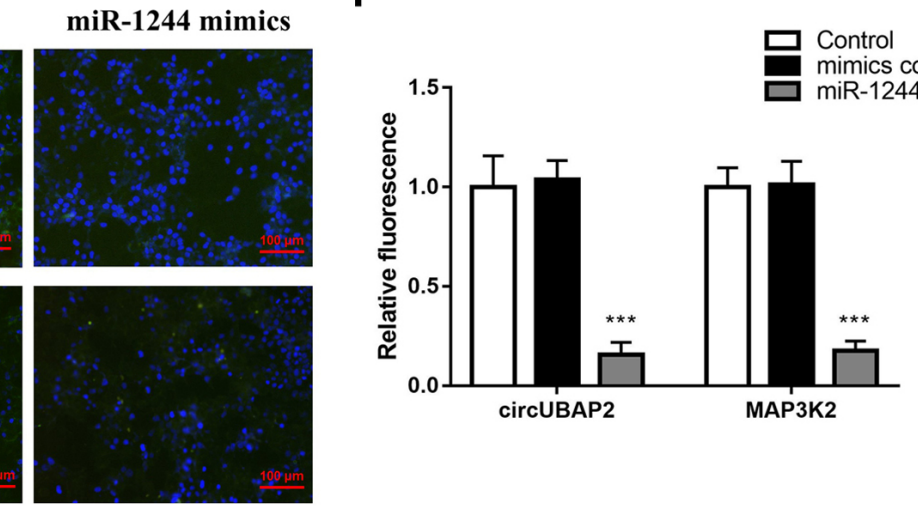

Figure 3. Validation of PCa-associated circUBAP2/miR-1244/MAP3K2 axis. (A) MAP3K2 expression was significantly upregulated following miR-1244-knockdown. (B) Putative binding sites of miR-1244 on circUBAP2 and MAP3K2. (C) Expression levels of circUBAP2, miR-1244 and MAP3K2 in clinical PCa and normal tissues. (D) Correlation between circUBAP2, miR-1244 and MAP3K2 expression in clinical tissues of PCa. (E and F) Fluorescence activity was decreased in 293T cells co-transfected with circUBAP2/MAP3K2 reporter plasmid (pGL6-miR plasmid) and miR-1244 mimics. **P $<0.01$ and ${ }^{* * * *} \mathrm{P}<0.001$ vs. control. PCa, prostate cancer; circUBAP2, circular RNA UBAP2; miR, microRNA; UTR, untranslated region.

based on the miRNA expression dataset GSE76260, which included 172 dysregulated miRNAs; however, only miR-1244 was found to be differentially expressed in PCa (Fig. 2C). Subsequently, the target mRNAs of miR-1244 were predicted using miRDB, TargetScan, miRWalk and TargetMiner. A total of 22 mRNAs were co-predicted as potential target mRNAs of circUBAP2/miR-1244 (Fig. 2D). Among these mRNAs, adducin 3 (ADD3), dynein cytoplasmic 1 intermediate chain 1 (DYNC1I1), MAP3K2 and Rho GTPase activating protein 20 (ARHGAP20) were found to be differentially expressed (upregulated) in the mRNA expression profile GSE30994 (Fig. 2E). Finally, a ceRNA network was constructed to assess the association of circUBAP2, miR-1244, ADD3, DYNC1I1, MAP3K2 and ARHGAP20 (Fig. 2F).

Validation of PCa-associated circUBAP2/miR-1244/MAP3K2 axis. In the present study, miR-1244 expression was knocked down using a miR-1244 inhibitor to validate its target mRNAs in PCa cells. Only MAP3K2 expression was significantly upregulated following miR-1244-knockdown, indicating that MAP3K2 was the final target mRNA of the circUBAP2/miR-1244 axis (Fig. 3A). Additionally, their potential binding sites were validated using TargetScan (Fig. 3B). Subsequently, the expression levels of circUBAP2, miR-1244 
A

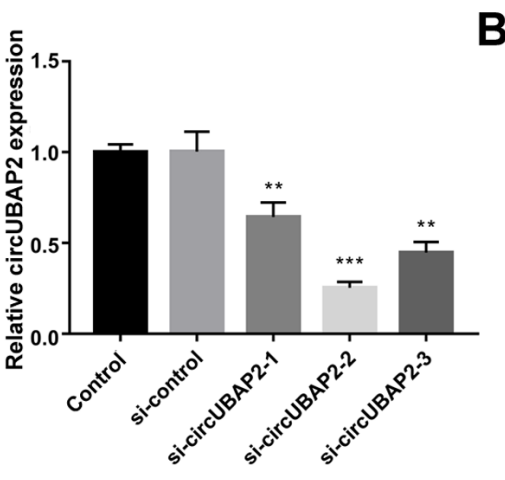

D

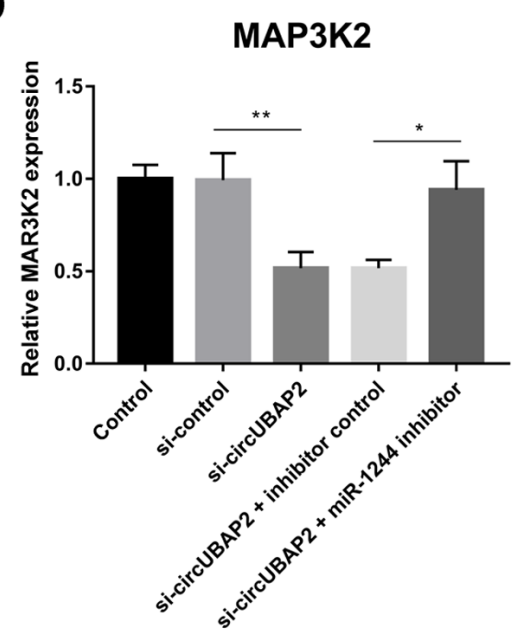

JNK

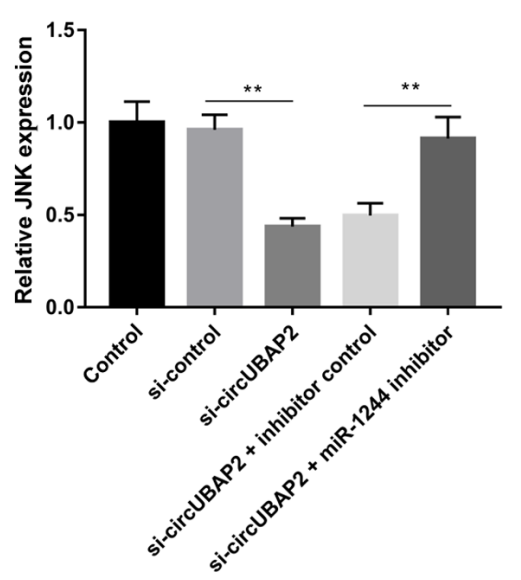

B

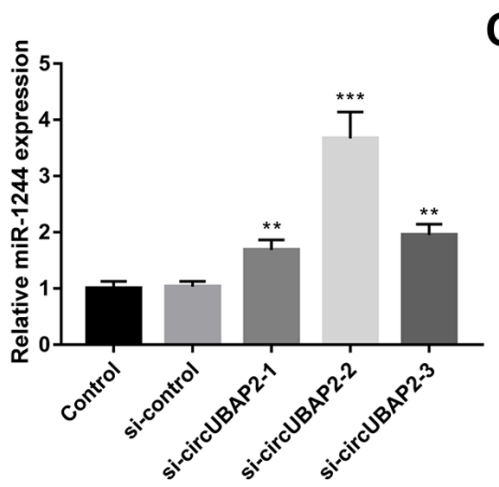

ERK
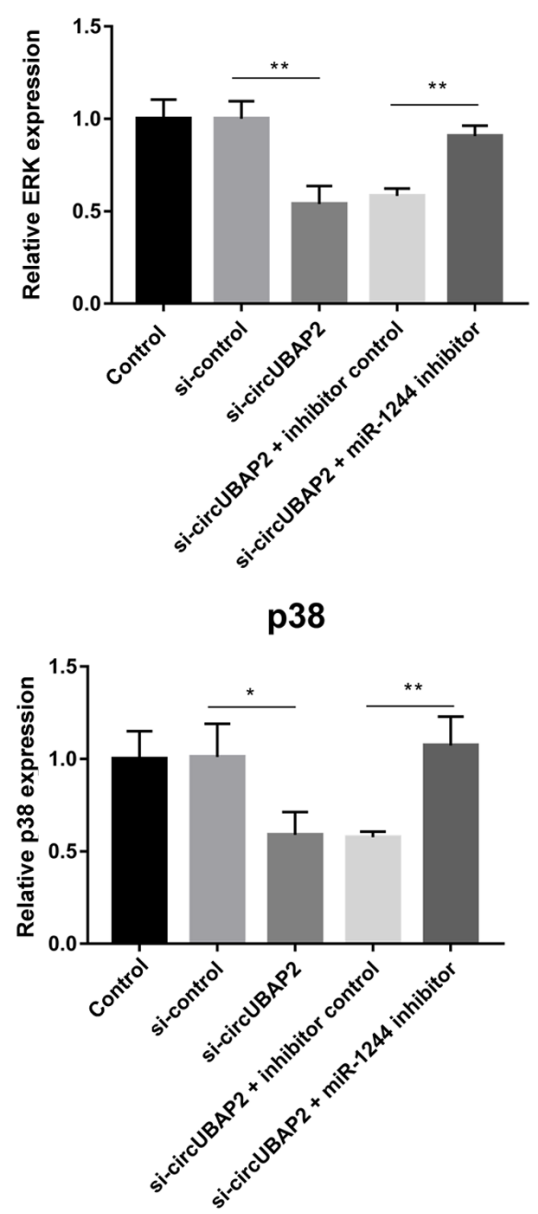

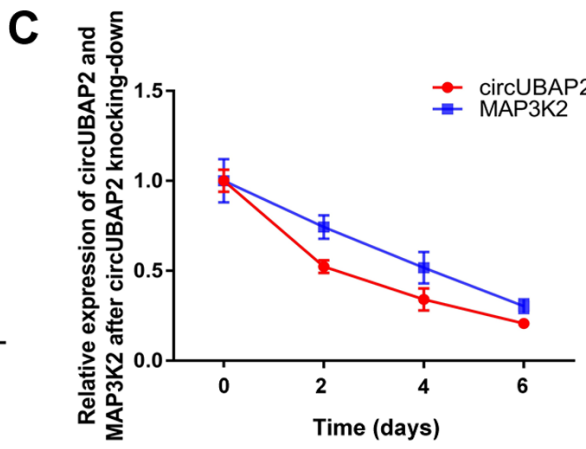

$\mathbf{E}$

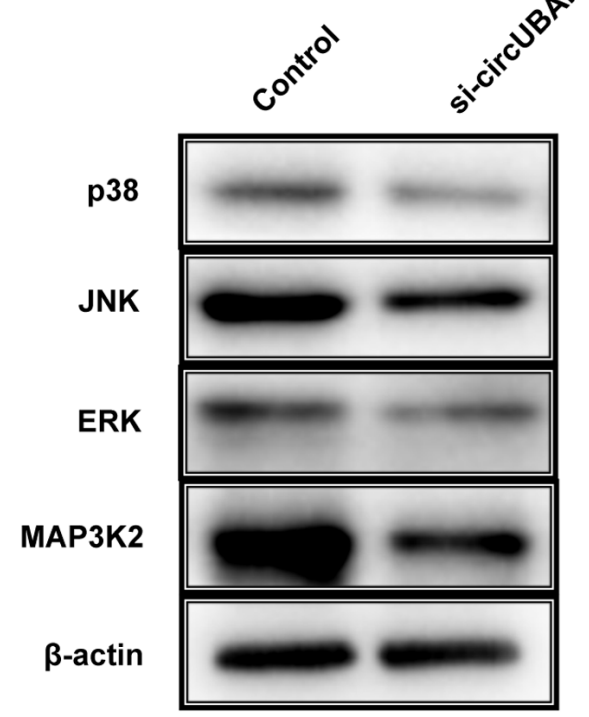

Figure 4. Functional validation of the circUBAP2/miR-1244/MAP3K2 axis. si-circUBAP2-2 exhibited the most significant effect on (A) knocking down circUBAP2 expression and (B) increasing miR-1244 expression. (C) MAP3K2 expression was decreased after si-circUBAP2 transfection and was positively associated with circUBAP2 expression. (D) Expression of MAP3K2 and MAPK key factors (ERK, JNK and p38) were significantly decreased after circUBAP2-knockdown and were then reversed by co-transfection with the miR-1244 inhibitor. (E) Western blot assay indicated that the protein expression levels of MAP3K2, ERK, JNK and p38 were decreased after si-circUBAP2 transfection. ${ }^{*} \mathrm{P}<0.05,{ }^{* *} \mathrm{P}<0.01$ and ${ }^{* * *} \mathrm{P}<0.001$ vs. control. circUBAP2, circular RNA UBAP2; miR, microRNA; si, small interfering RNA.

and MAP3K2 were detected in tissues of patients with PCa. The results indicated that circUBAP2 and MAP3K2 expression was significantly upregulated, while miR-1244 expression was significantly downregulated in PCa tissues compared with in normal tissues (Fig. 3C). Furthermore, circUBAP2 expression was negatively correlated with miR-1244 expression (Fig. 3D; R=-0.9421; $\mathrm{P}<0.001$ ), but positively correlated with MAP3K2 expression (Fig. 3D; R=0.8583; P<0.001). Additionally, a negative correlation was observed between
miR-1244 and MAP3K2 expression (Fig. 3D; R=-0.9124; $\mathrm{P}<0.001)$. In addition, the expression of circUBAP 2 was partly inhibited by overexpression of miR-1244, but promoted by inhibition of miR-1244 (Fig. S1). The luciferase reporter gene assay was further performed to validate the binding sites of miR-1244 on circUBAP2 and MAP3K2. The results indicated that the luciferase activity was significantly decreased in $293 \mathrm{~T}$ cells co-transfected with the circUBAP2/MAP3K2 reporter plasmid (pGL6-miR plasmid) and miR-1244 mimics 

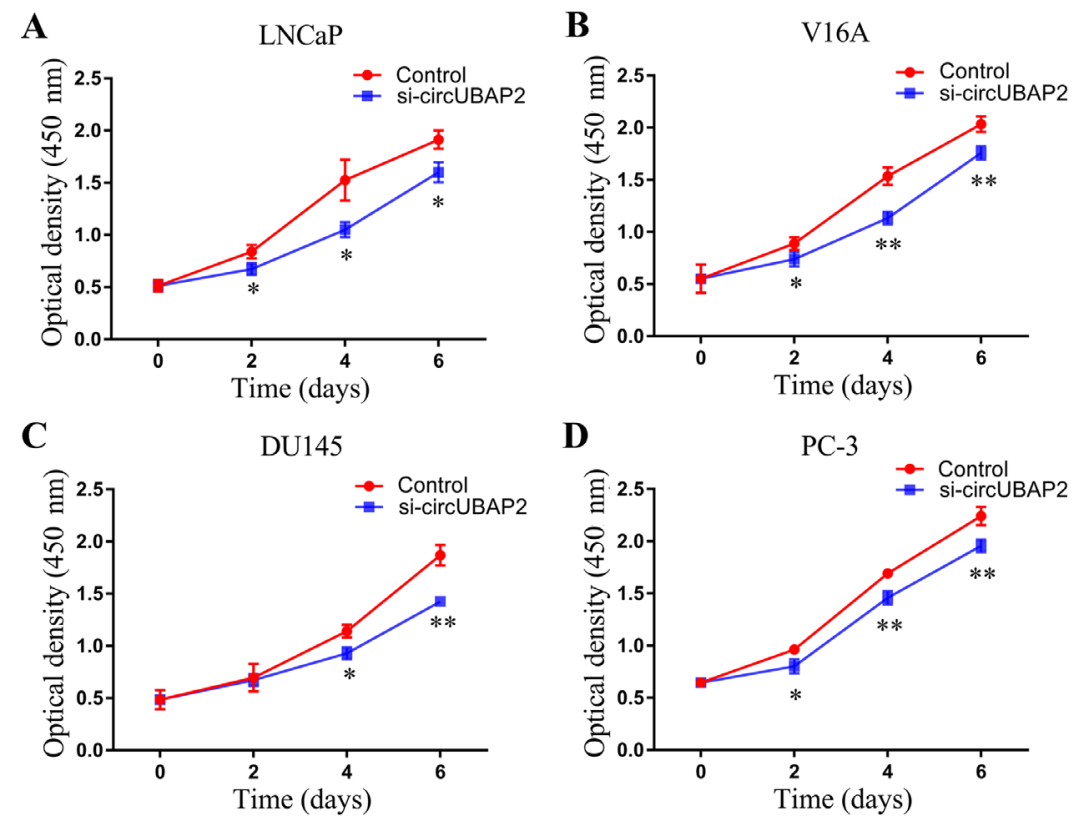

Figure 5. Proliferation of prostate cancer cells. Cell proliferation of (A) LNCaP, (B) V16A, (C) DU145 and (D) PC-3 cells was significantly decreased after circUBAP2-knockdown. ${ }^{*} \mathrm{P}<0.05$ and ${ }^{* * *} \mathrm{P}<0.01$ vs. control. circUBAP2, circular RNA UBAP2.

(Fig. S2A) compared with the other groups (Fig. 3E and F), while there was no difference between the circUBAP2 and MAP3K2 groups.

Functional verification of the circUBAP2/miR-1244/ MAP3K2 axis in LNCAP cells. As the LNCaP cell line presented the highest circUBAP2 expression, the regulating role of circUBAP2 was further explored in the $\mathrm{LNCaP}$ cell line. First, the effect of three si-circUBAP2 was examined on knocking down circUBAP2 expression, indicating that si-circUBAP2-2 had the most significant effect on knocking down circUBAP2 expression and increasing miR-1244 expression (Fig. 4A and B). Additionally, circUBAP2 expression was decreased in tandem with MAP3K2 expression (Fig. 4C). Subsequently, the miR-1244 inhibitor was used for rescue assays (Fig. S2B). The mRNA expression levels of MAP3K2, ERK, JNK and p38 were all significantly decreased $48 \mathrm{~h}$ after circUBAP2-knockdown (Fig. 4D). However, their expression levels were significantly reversed by co-transfection with the miR-1244 inhibitor (Fig. 4D). Since the mRNA expression of MAP3K2, ERK, JNK and p38 were significantly reversed by rescue experiments, which indicated that knocking down circUBAP2 could suppress the expression of these genes via targeting miR-1244. Hence, the inhibitory effects of knocking-down circUBAP2 on protein expression of MAP3K2, ERK, JNK and p38 were further validated. The western blotting results also confirmed that the protein expression levels of MAP3K2, ERK, JNK and p38 were decreased at day 6 (the most effective time point) after si-circUBAP2 transfection compared with the untransfected group (Fig. 4E).

Effect of circUBAP2 on cell proliferation of four PCa cell lines. The effect of circUBAP2 on the proliferation of $\mathrm{LNCaP}$ cells was measured using the CCK- 8 assay. The proliferation of LNCaP, V16A, DU145 and PC-3 cells was tested every 2 days after circUBAP2-knockdown. As shown in Fig. 5, the proliferative activity of all PCa cell lines was significantly decreased following circUBAP2-knockdown.

\section{Discussion}

circRNAs are critical regulators that serve an imperative role in gene expression $(14,27)$. circRNA biomarkers and circRNA-targeted therapy have attracted increasing attention in recent years $(28,29)$. Previous studies have revealed the differential expression profile of circRNAs in PCa, indicating that circRNAs may act as important factors in regulating the progression of PCa $(30,31)$. circUBAP2 is a well-known tumor-associated circRNA. Wu et al (16) indicated that circ-UBAP2 promoted the malignant behaviors of esophageal squamous cell carcinoma via the miR-422a/Rab10 axis. Zhao et al (17) found that circUBAP2 modulated pancreatic adenocarcinoma by regulating the infiltration and function of immune cells. Additionally, Sheng et al (15) identified circUBAP2 as a critical circRNA that promoted the progression of ovarian cancer by targeting miR-144. A preliminary study screened 171 circRNAs that were differentially expressed in PCa tissues, including circUBAP2 (upregulated) (18). However, the regulatory effect of circUBAP2 in the progression of PCa remains unclear.

Thus, the present study aimed to further explore the regulatory effect and mechanism of circUBAP2 in PCa. First, the expression levels of circUBAP2 were detected in four $\mathrm{PCa}$ cell lines, indicating that circUBAP2 expression was upregulated in PCa cells and that circUBAP2 was mainly located in the cytoplasm. Subsequently, site prediction websites were used to predict the targets of circUBAP2 in regulating the progression of PCa. Based on the miRNA expression profile GSE76260 and the mRNA expression profile GSE30994, it was determined that the circUBAP2/miR-1244/MAP3K2 axis may be critical for PCa progression. miR-1244 has been reported to act as a suppressor in various types of cancer, 
such as ovarian cancer and lung carcinoma by inhibiting its target mRNAs, such as pyruvate kinase M1/2 and myocyte enhancer factor 2D $(14,32,33)$. Moreover, the miRNA expression profile GSE76260 revealed that miR-1244 was downregulated in PCa. MAP3K2 is a stress-activated protein kinase in the MAPK signaling pathway, which is essential in promoting cancer cell proliferation, including in PCa $(23,34)$. However, the regulatory association between miR-1244 and MAP3K2 has not been fully defined. Notably, MAP3K2 expression in the present study was found to be upregulated in PCa tissues, which corresponded to the expression trend of circUBAP2, while it was negatively correlated with miR-1244 expression. Hence, it was hypothesized that the upregulation of circUBAP2 in PCa cells may suppress miR-1244 expression and promote MAP3K2 expression. High MAP3K2 expression may thereby promote cell proliferation of $\mathrm{PCa}$ cells.

To further prove this hypothesis, several lines of evidence were provided. First, the binding sites of miR-1244 on circUBAP2 and MAP3K2 were further confirmed by a luciferase reporter gene assay. Functional assays were then performed to verify the effect of circUBAP2 on the expression levels of MAP3K2 and MAPK key factors, including ERK, JNK and p38. circUBAP2-knockdown using si-circUBAP2 significantly decreased the expression levels of MAP3K2, ERK, JNK and p38 in LNCaP cells; their expression levels were then rescued by co-transfection with the miR-1244 inhibitor. Additionally, a CCK-8 assay was performed to verify the effect of circUBAP2 on the proliferation of $\mathrm{PCa}$ cells. The results revealed that circUBAP2-knockdown significantly decreased cell proliferation, indicating that circUBAP2 may have a role in promoting cell proliferation. Overall, the current data suggested that circUBAP2 may promote the proliferation of PCa cells via the miR-1244/ MAP3K2 axis.

In summary, the present study indicated that the circUBAP2/miR-1244 MAP3K2 axis may promote the proliferation of PCa cells, since circUBAP2-knockdown suppressed cell proliferation and the progression of $\mathrm{PCa}$. These findings may be essential in providing new therapeutic strategies for PCa.

\section{Acknowledgements}

Not applicable.

\section{Funding}

The present study was supported by the Department of Science and Technology of Xinjiang Uygur Autonomous Region, China (grant no. 2017DOIC294).

\section{Availability of data and materials}

The datasets used and/or analyzed during the current study are available from the corresponding author on reasonable request. The GSE76260 (https://www.ncbi.nlm.nih.gov/geo/query/acc. cgi?acc=GSE76260) and GSE30994 (https://www.ncbi.nlm. nih.gov/geo/query/acc.cgi) datasets are available in the Gene Expression Omnibus repository.

\section{Authors' contributions}

XL and YW designed and supervised the study, as well as organized and wrote the manuscript. XL, BA and WW performed the data analysis. XL, MR, and CX contributed to acquisition of data. XL, BA, WW, MR, CX and YW confirm the authenticity of the raw data. All authors read and approved the final manuscript.

\section{Ethics approval and consent to participate}

Written informed consent was obtained from all participants. All study protocols were approved by the Ethics Committee of The First Affiliated Hospital of Xinjiang Medical University (approval no. XJ20200025).

\section{Patient consent for publication}

Not applicable.

\section{Competing interests}

The authors declare that they have no competing interests.

\section{References}

1. Szentirmai E and Giannico GA: Intraductal carcinoma of the prostate. Pathologica 112: 17-24, 2020.

2. Xin L: Cells of origin for prostate cancer. Adv Exp Med Biol 1210: 67-86, 2019.

3. Sartor O and de Bono JS: Metastatic prostate cancer. N Engl J Med 378: 645-657, 2018.

4. Abrams DI: An integrative approach to prostate cancer. J Altern Complement Med 24: 872-880, 2018.

5. Merriel SWD, Funston G and Hamilton W: Prostate cancer in primary care. Adv Ther 35: 1285-1294, 2018.

6. Zhou CK, Check DP, Lortet-Tieulent J, Laversanne M, Jemal A, Ferlay J, Bray F, Cook MB and Devesa SS: Prostate cancer incidence in 43 populations worldwide: An analysis of time trends overall and by age group. Int J Cancer 138: 1388-1400, 2016.

7. Pang C, Guan Y, Li H, Chen W and Zhu G: Urologic cancer in China. Jpn J Clin Oncol 46: 497-501, 2016.

8. Shan X, Danet-Desnoyers G, Aird F, Kandela I, Tsui R, Perfito N and Iorns E: Replication study: Androgen receptor splice variants determine taxane sensitivity in prostate cancer. PeerJ 6: e4661, 2018.

9. Kristensen LS, Andersen MS, Stagsted L, Ebbesen KK, Hansen TB and Kjems J: The biogenesis, biology and characterization of circular RNAs. Nat Rev Genet 20: 675-691, 2019.

10. Chen G, Wang Q, Li Z, Yang Q, Liu Y, Du Z, Zhang G and Song Y: Circular RNA CDR1as promotes adipogenic and suppresses osteogenic differentiation of BMSCs in steroid-induced osteonecrosis of the femoral head. Bone 133: 115258, 2020.

11. Wu P, Mo Y, Peng M, Tang T, Zhong Y, Deng X, Xiong F, Guo C, Wu X, Li Y, et al: Emerging role of tumor-related functional peptides encoded by lncRNA and circRNA. Mol Cancer 19: 22, 2020.

12. Zhang Q, Wang W, Zhou Q, Chen C, Yuan W, Liu J, Li X and Sun Z: Roles of circRNAs in the tumour microenvironment. Mol Cancer 19: 14, 2020

13. Tang $Q$ and Hann SS: Biological roles and mechanisms of circular RNA in human cancers. Onco Targets Ther 13: 2067-2092, 2020.

14. Lei M, Zheng G, Ning Q, Zheng J and Dong D: Translation and functional roles of circular RNAs in human cancer. Mol Cancer 19: 30, 2020.

15. Sheng M, Wei N, Yang HY, Yan M, Zhao QX and Jing LJ: CircRNA UBAP2 promotes the progression of ovarian cancer by sponging microRNA-144. Eur Rev Med Pharmacol Sci 23: 7283-7294, 2019.

16. Wu Y, Zhi L, Zhao Y, Yang L and Cai F: Knockdown of circular RNA UBAP2 inhibits the malignant behaviours of esophageal squamous cell carcinoma by microRNA-422a/Rab10 axis. Clin Exp Pharmacol Physiol 47: 1283-1290, 2020. 
17. Zhao R, Ni J, Lu S, Jiang S, You L, Liu H, Shou J, Zhai C, Zhang W, Shao S, et al: CircUBAP2-mediated competing endogenous RNA network modulates tumorigenesis in pancreatic adenocarcinoma. Aging (Albany NY) 11: 8484-8501, 2019.

18. Chen S, Huang V, Xu X, Livingstone J, Soares F, Jeon J, Zeng Y, Hua JT, Petricca J, Guo H, et al: Widespread and functional RNA circularization in localized prostate cancer. Cell 176: 831-843. e22, 2019.

19. Dizeyi N, Hedlund P, Bjartell A, Tinzl M, Austild-Taskén K and Abrahamsson PA: Serotonin activates MAP kinase and $\mathrm{PI} 3 \mathrm{~K} /$ Akt signaling pathways in prostate cancer cell lines. Urol Oncol 29: 436-445, 2011.

20. Pan C, Zhang L, Meng X, Qin H, Xiang Z, Gong W, Luo W, Li D and Han X: Chronic exposure to microcystin-LR increases the risk of prostate cancer and induces malignant transformation of human prostate epithelial cells. Chemosphere 263: $128295,2021$.

21. Livak KJ and Schmittgen TD: Analysis of relative gene expression data using real-time quantitative PCR and the 2(-Delta Delta C(T)) method. Methods 25: 402-408, 2001

22. Kim J, Mizokami A, Shin M, Izumi K, Konaka H, Kadono Y, Kitagawa Y, Keller ET, Zhang J and Namiki M: SOD3 acts as a tumor suppressor in PC-3 prostate cancer cells via hydrogen peroxide accumulation. Anticancer Res 34: 2821-2831, 2014.

23. Wang LL and Zhang M: miR-582-5p is a potential prognostic marker in human non-small cell lung cancer and functions as a tumor suppressor by targeting MAP3K2. Eur Rev Med Pharmacol Sci 22: 7760-7767, 2018

24. Chen G, Wang Q, Yang Q, Li Z, Du Z, Ren M, Zhao H, Song Y and Zhang G: Circular RNAs hsa_circ_0032462, hsa_circ_0028173, hsa_circ_0005909 are predicted to promote CADM1 expression by functioning as miRNAs sponge in human osteosarcoma. PLoS One 13: e0202896, 2018.

25. Wang Q, Yang Q, Chen G, Du Z, Ren M, Wang A, Zhao H, Li Z, Zhang $G$ and Song Y: LncRNA expression profiling of BMSCs in osteonecrosis of the femoral head associated with increased adipogenic and decreased osteogenic differentiation. Sci Rep 8 9127,2018
26. Dai J, Zhuang Y, Tang M, Qian Q and Chen JP: CircRNA UBAP2 facilitates the progression of colorectal cancer by regulating miR-199a/VEGFA pathway. Eur Rev Med Pharmacol Sci 24: 7963-7971, 2020

27. Sulaiman SA, Abdul Murad NA, Mohamad Hanif EA, Abu N and Jamal R: Prospective advances in circular RNA investigation. Adv Exp Med Biol 1087: 357-370, 2018.

28. Arnaiz E, Sole C, Manterola L, Iparraguirre L, Otaegui D and Lawrie CH: CircRNAs and cancer: Biomarkers and master regulators. Semin Cancer Biol 58: 90-99, 2019.

29. Holdt LM, Kohlmaier A and Teupser D: Circular RNAs as therapeutic agents and targets. Front Physiol 9: 1262, 2018.

30. Hua JT, Chen S and He HH: Landscape of noncoding RNA in prostate cancer. Trends Genet 35: 840-851, 2019.

31. Yan Z, Xiao Y, Chen Y and Luo G: Screening and identification of epithelial-to-mesenchymal transition-related circRNA and miRNA in prostate cancer. Pathol Res Pract 216: 152784, 2020.

32. Liu Y, He X, Chen Y and Cao D: Long non-coding RNA LINC00504 regulates the Warburg effect in ovarian cancer through inhibition of miR-1244. Mol Cell Biochem 464: 39-50, 2020.

33. Zhang R, Zhang Y and Li H: miR-1244/myocyte enhancer factor 2D regulatory loop contributes to the growth of lung carcinoma. DNA Cell Biol 34: 692-700, 2015.

34. Lv X, Wang M, Qiang J and Guo S: Circular RNA circ-PITX1 promotes the progression of glioblastoma by acting as a competing endogenous RNA to regulate miR-379-5p/MAP3K2 axis. Eur J Pharmacol 863: 172643, 2019.

This work is licensed under a Creative Commons Attribution-NonCommercial-NoDerivatives 4.0 International (CC BY-NC-ND 4.0) License. 